\title{
PENELOPE/PRIMO-calculated photon and electron spectra from clinical accelerators
}

\author{
Lorenzo Brualla $1,2,3,4^{*}$ (D) Miguel Rodriguez ${ }^{5,6}$, Josep Sempau ${ }^{7}$ and Pedro Andreo ${ }^{8}$
}

\begin{abstract}
Background: The availability of photon and electron spectra in digital form from current accelerators and Monte Carlo (MC) systems is scarce, and one of the packages widely used refers to linacs with a reduced clinical use nowadays. Such spectra are mainly intended for the MC calculation of detector-related quantities in conventional broad beams, where the use of detailed phase-space files (PSFs) is less critical than for MC-based treatment planning applications, but unlike PSFs, spectra can easily be transferred to other computer systems and users.

Methods: A set of spectra for a range of Varian linacs has been calculated using the PENELOPE/PRIMO MC system. They have been extracted from PSFs tallied for field sizes of $10 \mathrm{~cm} \times 10 \mathrm{~cm}$ and $15 \mathrm{~cm} \times 15 \mathrm{~cm}$ for photon and electron beams, respectively. The influence of the spectral bin width and of the beam central axis region used to extract the spectra have been analyzed.

Results: Spectra have been compared to those by other authors showing good agreement with those obtained using the, now superseded, EGS4/BEAM MC code, but significant differences with the most widely used photon data set. Other spectra, particularly for electron beams, have not been published previously for the machines simulated in this work. The influence of the bin width on the spectrum mean energy for 6 and $10 \mathrm{MV}$ beams has been found to be negligible. The size of the region used to extract the spectra yields differences of up to $40 \%$ for the mean energies in $10 \mathrm{MV}$ beams, but the maximum difference for TPR20,10 values derived from depth-dose distributions does not exceed $2 \%$ relative to those obtained using the PSFs. This corresponds to $k_{Q}$ differences below $0.2 \%$ for a typical Farmer-type chamber, considered to be negligible for reference dosimetry. Different configurations for using electron spectra have been compared for $6 \mathrm{MeV}$ beams, concluding that the geometry used for tallying the PSFs used to extract the spectra must be accounted for in subsequent calculations using the spectra as a source.
\end{abstract}

Conclusions: An up-to-date set of consistent spectra for Varian accelerators suitable for the calculation of detector-related quantities in conventional broad beams has been developed and made available in digital form.

Keywords: Monte Carlo, PENELOPE, penEasy, PRIMO, Linac spectra, Radiotherapy physics, Radiotherapy dosimetry, Radiotherapy accelerators

\section{Introduction}

It is well-known that a comprehensive phase-space file (PSF) characterizing the energy, position, direction and statistical weight of all the particle generations emerging from a clinical accelerator and reaching the surface of a phantom or a patient, provides a suitable source for Monte Carlo (MC) radiotherapy dosimetry calculations. These include the calculation of detector-related quantities in

*Correspondence: Iorenzo.brualla@uni-duisburg-essen.de

${ }^{1}$ West German Proton Therapy Centre Essen (WPE), D-45147 Essen, Germany

${ }^{2}$ West German Cancer Center (WTZ), D-45147 Essen, Germany

Full list of author information is available at the end of the article and patient treatment planning (see references [1, 2]). PSFs should include a very large number of particles to minimize as much as possible the so-called latent variance [3] in the calculated quantity, although strictly the statistical variance is only part of the simulated story, since it should be combined with the type A uncertainty of the MC calculation. Hence, PSFs are usually very large, of the order of gigabytes (GB), their size being an inconvenient at the time of a data transfer or exchange among users.

PSFs are necessary for MC simulations involving the extended 3-D geometries of patient CT data, but the

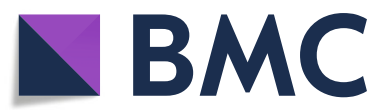

(c) The Author(s). 2019 Open Access This article is distributed under the terms of the Creative Commons Attribution 4.0 International License (http://creativecommons.org/licenses/by/4.0/), which permits unrestricted use, distribution, and reproduction in any medium, provided you give appropriate credit to the original author(s) and the source, provide a link to the Creative Commons license, and indicate if changes were made. The Creative Commons Public Domain Dedication waiver (http://creativecommons.org/publicdomain/zero/1.0/) applies to the data made available in this article, unless otherwise stated. 
necessity can be relaxed to some extent for the simulation of detector-related quantities, as those included in dosimetry protocols [4-8], since equivalent results are obtained [9]. For the latter, using the "main incident particle" spectrum, i.e., photons or electrons, has become the most common practice. It should be noticed that spectra extracted from a small central region of a broad beam is not equivalent to that obtained from the simulation of a small beam.

The most widely used set of clinical accelerator photon spectra for conventional broad beams is that calculated by Mohan et al. [10], henceforth referred to as Mohan, for four Varian Clinac machines with acceleration potentials between $4 \mathrm{MV}$ and $24 \mathrm{MV}$ using the EGS4 MC system [11]. A reason for its frequent use is that the set of spectra is included in digital form in the EGSnrc [12] distribution package since long ago. A set of nine photon broad beam spectra from accelerators manufactured by Elekta, Siemens and Varian was published by Sheikh-Bagheri and Rogers [13], henceforth referred to as SBR; they were also calculated with the EGS4 MC system, using its BEAM code [14]. These spectra, unlike those of Mohan, were presented in tabular form with a bin width of $250 \mathrm{keV}$; however, they were not included in the EGSnrc package and, probably, due to this reason their use has been rather limited.

Electron broad beam spectra have, on the other hand, received much lower attention, to the extent that no detailed tabulation has been made available in the peerreviewed literature. An internal report by Ding and Rogers [15], henceforth referred to as DR, included a large set of electron spectra in the energy range of 5-50 MeV from various accelerators. They were also calculated with the EGS4/BEAM code and presented in graphical form, but despite its introductory statement on digital availability, neither the report nor the data can be found in the Internet address provided or in the EGSnrc or the National Research Council of Canada web sites. The set is not included either in the current EGSnrc package.

The IAEA has also developed a database of PSFs [16] that includes ${ }^{60} \mathrm{Co} \gamma$ rays and a number of accelerator photon and electron beams (https:// www-nds.iaea.org/phsp) from where spectra can be extracted, but the range of beam energies available is rather limited. The database has not been updated since 2013.

Considering that many of the photon and electron spectra mentioned are from accelerators no longer in clinical use, and the general lack of availability of their data in digital form, a project was undertaken to produce a consistent set of the two types of spectra for conventional broad beams from current accelerators. The spectra are extracted from PSFs calculated with the MC system PENELOPE/PRIMO [17-19]. This work provides a description of the methods and calculations performed to derive the data and provides tables of spectra for 6-22 $\mathrm{MeV}$ electron beams and 6-20 MV photon beams, the latter group including two flattening-filter-free (FFF) beams in frequent clinical use. The work also includes the analysis of the influence of the spectral bin size and the size of the region around the beam central axis used to



Fig. 1 Calculated photon spectra (planar fluence differential in energy) for 6, 10, 15 and 20 MV Varian clinical beams normalized to their integral. The dashed histograms correspond to spectra for 6 and 10 MV FFF beams. Observe the 511 keV peak resulting from positron annihilation, visible in the higher energy beams 


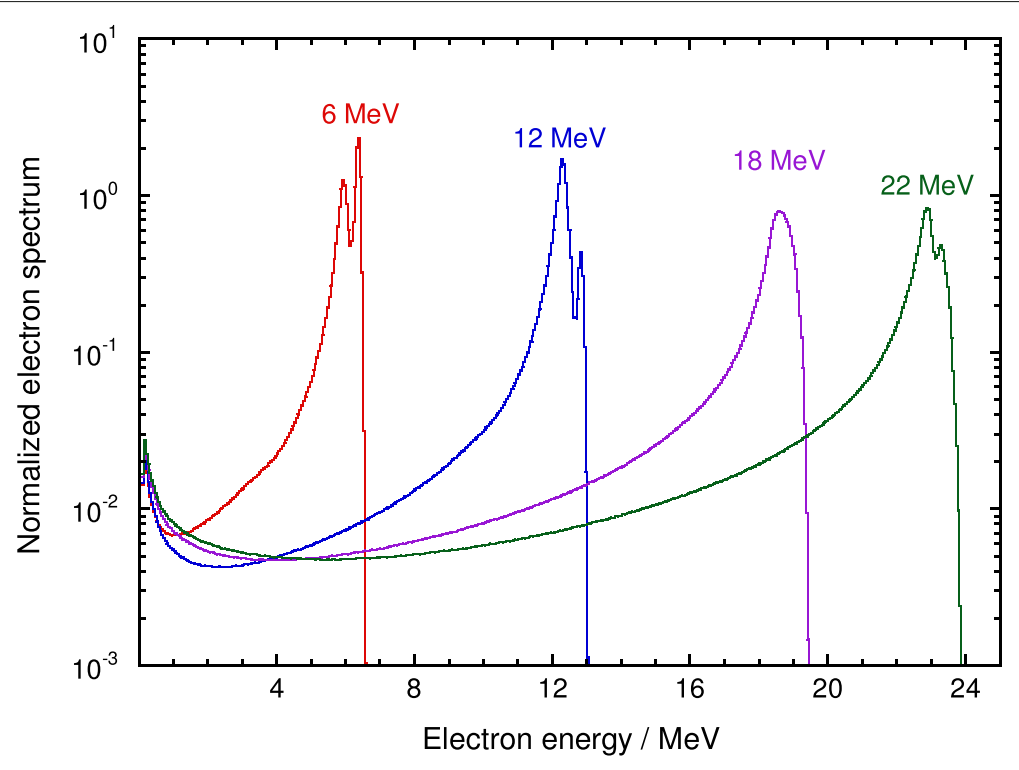

Fig. 2 Calculated electron spectra (planar fluence differential in energy) for 6, 12, 18 and 22 MeV Varian clinical beams normalized to their integral

extract the spectra for 6 and $10 \mathrm{MV}$. Comparisons with other published spectra are made whenever data for the same or similar linacs are available. The spectra in digital form are available in the PRIMO web site (https://www. primoproject.net).

\section{Material and methods}

The calculations presented in this work have been performed with the PRIMO software (version 0.3.1.1681)
$[18,19]$, which is based on the PENELOPE (version 2011) / penEasy Monte Carlo code.

PENELOPE [20] is a general-purpose MC system for the simulation of the transport of photons and electrons in arbitrary media. Its early use for simulating linac electron beams goes back to 2001 [3]. PENELOPE provides the calculation engine for a number of codes developed for the simulation of the treatment head of different accelerators, such as PENLINAC [21],

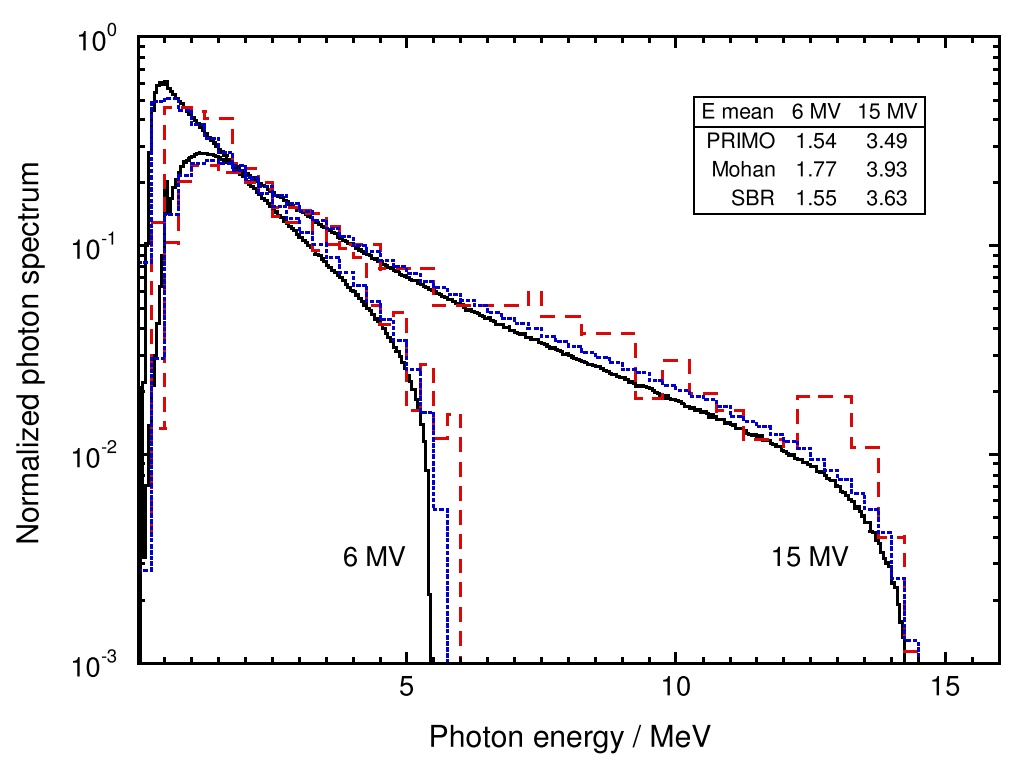

Fig. 3 Comparison of the PENELOPE/PRIMO photon spectra for Varian beams of 6 and 15 MV with those calculated by Mohan et al. [10], dashed histograms, and Sheikh-Bagheri and Rogers [13], dotted histograms. All the spectra are normalized to their respective integral. The fluence-weighted mean energies of each spectrum are indicated in the inset 
PENEASYLINAC [22] and PRIMO. PENELOPE is in fact a routine library which requires a steering main program to provide, among other aspects, the description of the particle source, the tallies to be scored and certain variance-reduction techniques (VRT) that are applied at the main program level. The penEasy code [22] is one of such main programs for PENELOPE, which includes the handling of voxelized geometries. PRIMO is a free-software package that simulates clinical linacs and estimates absorbed dose distributions in phantoms and computerized tomographies; it combines a graphical user interface with the PENELOPE/penEasy system.
The spectra presented in this work have been extracted from PSFs tallied with PRIMO. Flattening filtered photon beams of 6, 10, 15 and 20 MV from a Varian Clinac C series (e.g., models 2100, 18, 1800, 2300 and iX) were simulated with a field size of $10 \mathrm{~cm} \times 10 \mathrm{~cm}$. FFF photon beams of 6 and $10 \mathrm{MV}$ from a Varian TrueBeam were simulated using the FakeBeam empirical geometry described by Rodriguez et al. [23], also with a field size of $10 \mathrm{~cm} \times 10 \mathrm{~cm}$. Electron beams of 6,12, 18 and $22 \mathrm{MeV}$ from the same Clinac $\mathrm{C}$ series were simulated with a field size of $15 \mathrm{~cm} \times 15 \mathrm{~cm}$ collimated with the electron applicator. The extracted spectra, averaged over either the entire beam area or restricted to a narrow


Fig. 4 Photon spectra for 6 and 10 MV beams, derived from the PSF for a $10 \mathrm{~cm} \times 10 \mathrm{~cm}$ field, for a central circular area of $2 \mathrm{~cm}$ diameter and a square of $2 \mathrm{~cm}$ side using bin widths of $50 \mathrm{keV}$, and for a square of $10 \mathrm{~cm}$ side using bin widths of 1,50 and $250 \mathrm{keV}$. All the spectra are normalized to their respective integral. Note the energy logarithmic scale for better visualization 
region around the beam central axis, were tallied in $250 \mathrm{keV}$-, $50 \mathrm{keV}$ - and $1 \mathrm{keV}$-wide bins, depending on the case.

The number of incident particles simulated was $10^{9}$ and $10^{10}$ for photon and electron beams, respectively, leading to PSFs of the order of $10^{8}-10^{9}$ particles. The size of the PSFs were 60-360 GB for the electron beams and 4-64 GB for photons. The speed and accuracy of the particle MC simulation was controlled using the following transport parameters:

(i) Particle cut-off energies, below which the transport of particles is halted, was set to $200 \mathrm{keV}$ for electrons and positrons, and to $50 \mathrm{keV}$ for photons.

(ii) The limits between detailed and condensed simulation of charged particles are governed in PENELOPE by $W_{\mathrm{CC}}, W_{\mathrm{CR}}, C_{1}$ and $C_{2}$. The first two parameters set the limit of the energy loss thresholds separating hard and soft events for electronic collisions and bremsstrahlung emission, respectively. $C_{1}$ and $C_{2}$ are related with the corresponding angular threshold for elastic deflections (refer to the PENELOPE manual for further details). For electron beams their values were $W_{\mathrm{CC}}=200 \mathrm{keV}$, $W_{\mathrm{CR}}=50 \mathrm{keV}$ and $C_{1}=C_{2}=0.1$. For photon beams the same transport parameters were used with the exception of $W_{\mathrm{CR}}=200 \mathrm{keV}$ for all materials and $C_{1}=C_{2}=0.001$ in the bremsstrahlung accelerator target.

(iii) The parameter DSMAX, defining the maximum step length allowed for electrons and positrons, was set to $1 / 10$ of the thickness of each component of the linac.

(iv) All the primary particle sources (for both electron and photon beams) were modeled as monoenergetic point sources with zero divergence.

(v) The accelerator incident electron energies were those set as default in PRIMO, which are a good ansatz for most Varian Clinac $C$ series machines. For electron beams, the energies used were $6.85 \mathrm{MeV}$ (for $6 \mathrm{MeV}$ ), 13.37 MeV (for $12 \mathrm{MeV}$ ), 19.97 MeV (for $18 \mathrm{MeV}$ ) and $24.46 \mathrm{MeV}$ (for $22 \mathrm{MeV}$ ). For photon beams they were $5.4 \mathrm{MeV}$ (for $6 \mathrm{MV}$ ), $10.5 \mathrm{MeV}$ (for $10 \mathrm{MV}$ ), $14.3 \mathrm{MeV}$ (for $15 \mathrm{MV}$ ) and $18.5 \mathrm{MeV}$ (for $20 \mathrm{MV}$ ).

(vi) The variance reduction techniques used to speed up the photon simulations were splitting roulette [24] for 6 and $10 \mathrm{MV}$, and rotational splitting [25] for 15 and $20 \mathrm{MV}$. No VRTs were used for the simulation of electron beams.

(vii) Electron PSFs were tallied downstream of the third scrapper of the electron applicator defining the $15 \mathrm{~cm} \times 15 \mathrm{~cm}$ field size at the phantom surface. Photon PSFs were tallied at the exit of the gantry for a $10 \mathrm{~cm} \times 10 \mathrm{~cm}$ field size at the phantom surface. The field size was defined at isocenter distance, as usual.

\section{Results and discussion}

The PSF-extracted spectra (strictly, planar fluence differential in energy [8]) for the photon and electron beams are shown in Figs. 1 and 2, respectively, where, to enable comparison, they have been normalized to their respective integrals. To facilitate the visualization of the spectra, uncertainties are not shown in the plot but the smoothness of the data indicates a rather low statistical uncertainty (type A). The spectra are given in numerical form in the Additional files 1 and 2, which includes the standard uncertainty of the fluence in each bin as a percentage of the value.

The bin width of the spectra shown in Figs. 1 and 2 is $50 \mathrm{keV}$. This width allows the visualization of $511 \mathrm{keV}$ photons resulting from positron annihilation, which are visible in the higher energy beams of Fig. 1, but would be almost unnoticeable using a broader width (see, e.g., figure 10 in SBR [13], where a bin width of $250 \mathrm{keV}$ was used).

Our calculated photon spectra are compared with those of Mohan [10] and SBR [13] for Varian broad beams of 6 and $15 \mathrm{MV}$ in Fig. 3, where the mean energies of the different spectra are indicated. These authors derived the spectra from a broad beam while recording the particles in a small central region of radii $3 \mathrm{~cm}$ and $2.25 \mathrm{~cm}$, respectively, whereas in the present work the spectra are for a $10 \mathrm{~cm} \times 10 \mathrm{~cm}$ field in which in the indicated cases particles from the whole field are recorded; this alternative should make their beams slightly harder than ours (see, e.g., refs. $[5,26])$. There are large differences in shape with Mohan's spectra. Additionally, their broad bin widths show a rather large uncertainty, and the mean energies differ from those in the present work by $15 \%$ (6 MV) and $12.6 \%$ (15 MV). The PRIMO spectra do not differ substantially from those of SBR [13] although slightly higher mean energies can be observed in consistency with the comment above regarding small fields; despite their broader bin width $(250 \mathrm{keV}$ versus our $50 \mathrm{keV})$ the mean energies differ by only $0.4 \%$ for the $6 \mathrm{MV}$ beam, while the difference

Table 1 Fluence-weighted $\left(\bar{E}_{\Phi}\right)$ and energy fluence-weighted $\left(\bar{E}_{\Psi}\right)$ mean energies of 6 and $10 \mathrm{MV}$ photon spectra for a central circular area of $2 \mathrm{~cm}$ diameter and a square of $2 \mathrm{~cm}$ side using bin widths of $50 \mathrm{keV}$, and for a square of $10 \mathrm{~cm}$ side using bin widths of 1,50 and $250 \mathrm{keV}$

\begin{tabular}{|c|c|c|c|c|c|}
\hline \multirow[b]{2}{*}{ Field size } & \multirow{2}{*}{$\begin{array}{l}\text { Bin width } \\
\text { (keV) }\end{array}$} & \multicolumn{2}{|l|}{$6 \mathrm{MV}$} & \multicolumn{2}{|l|}{$10 \mathrm{MV}$} \\
\hline & & $\overline{\bar{E}}_{\Phi}(\mathrm{MeV})$ & $\bar{E}_{\Psi}(\mathrm{MeV})$ & $\overline{\bar{E}}_{\Phi}(\mathrm{MeV})$ & $\bar{E}_{\Psi}(\mathrm{MeV})$ \\
\hline$\varnothing 2 \mathrm{~cm}$ & 50 & 1.604 & 2.456 & 3.000 & 4.664 \\
\hline$\square 2 \mathrm{~cm}$ side & 50 & 1.601 & 2.453 & 2.989 & 4.655 \\
\hline$\square 10 \mathrm{~cm}$ side & 50 & 1.542 & 2.398 & 2.760 & 4.471 \\
\hline$\square 10 \mathrm{~cm}$ side & 1 & 1.542 & 2.398 & 2.218 & 3.238 \\
\hline$\square 10 \mathrm{~cm}$ side & 250 & 1.542 & 2.401 & 2.760 & 4.473 \\
\hline
\end{tabular}

All the spectra are extracted from the PSF files for a $10 \mathrm{~cm} \times 10 \mathrm{~cm}$ field 
is $4.1 \%$ for $15 \mathrm{MV}$. Not shown in the plot to avoid cluttering, for $10 \mathrm{MV}$ the differences in mean energies are $7.5 \%$ for Mohan and $6.7 \%$ for SBR. It should also be noted that the differences mentioned, particularly in Mohan's case, could be related to changes in the linac modeling [27].

The influence of the region size around the central beam axis used to extract the photon spectrum from a $10 \mathrm{~cm} \times 10 \mathrm{~cm}$ PSF, and of the bin width of the spectrum have been investigated for 6 and $10 \mathrm{MV}$ photon spectra obtained for different conditions. These have been a circular area of $2 \mathrm{~cm}$ diameter and a square of $2 \mathrm{~cm}$ side for bin widths of $50 \mathrm{keV}$, and a square of $10 \mathrm{~cm}$ side for bin widths of 1,50 and $250 \mathrm{keV}$. The set of five spectra are shown in Fig. 4 for both beam nominal energies, and the corresponding fluence-weighted and energy fluence-weighted mean energies are given in Table 1.

It can be observed in the table that for the $6 \mathrm{MV}$ beams the dependence of the mean energy on the size of the extracting region is about $4 \%$ and $2 \%$ for $\bar{E}_{\Phi}$ and $\bar{E}_{\Psi}$, respectively, while the dependence on bin width is practically negligible. This is not the case, however, for the $10 \mathrm{MV}$ beam, where differences respect to the size of the
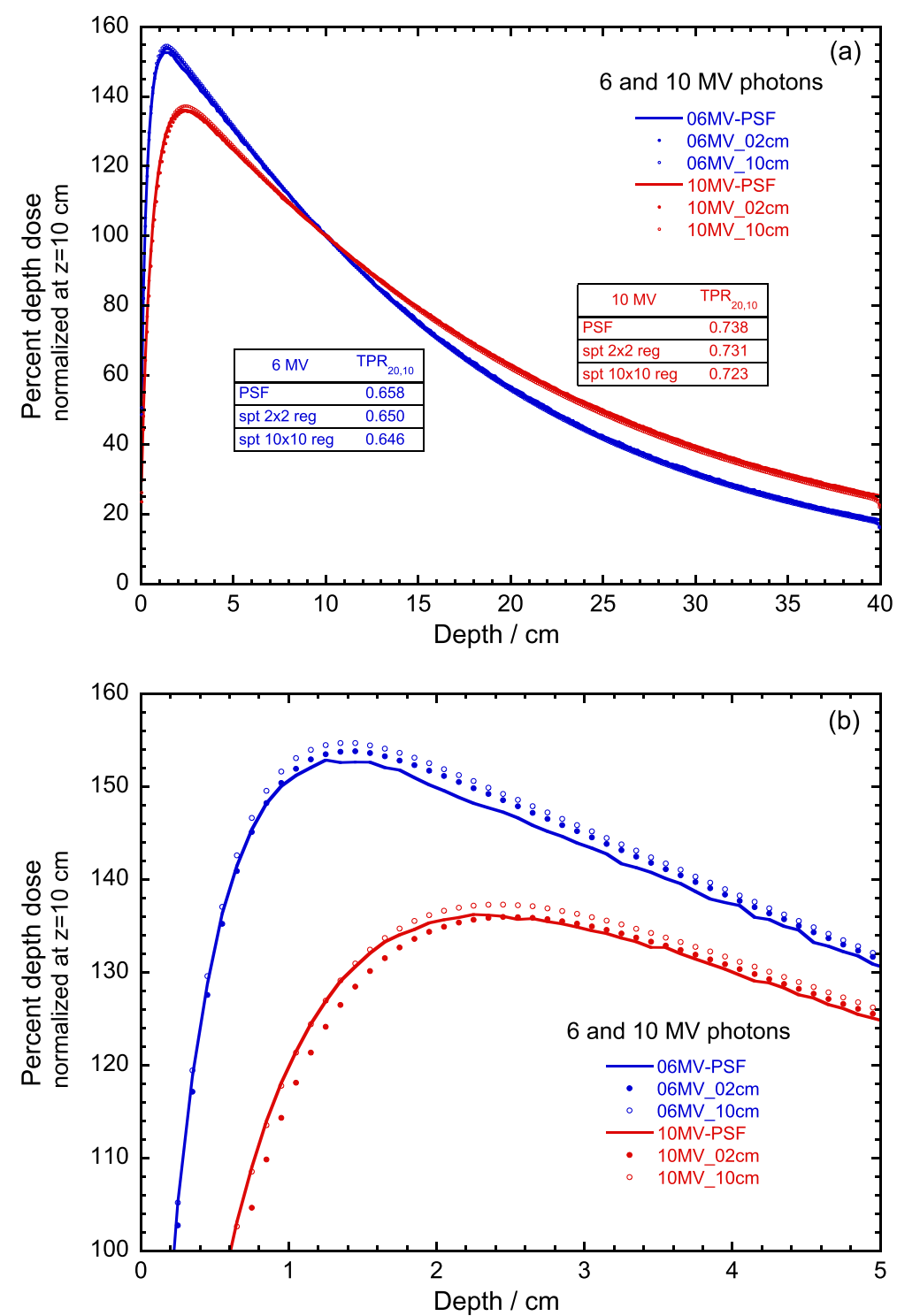

Fig. 5 (a) Comparison of 6 and $10 \mathrm{MV}$ photons depth-dose distributions, normalized at the depth of $10 \mathrm{~cm}$, for $10 \mathrm{~cm} \times 10 \mathrm{~cm}$ fields obtained from the PSFs (solid lines) with those obtained using the spectrum in a $2 \mathrm{~cm} \times 2 \mathrm{~cm}$ central region around the beam central axis (filled circles) and in $10 \mathrm{~cm} \times 10 \mathrm{~cm}$ (open circles). Panel (b) is an enlargement of the region around the maximum of the distributions. In all cases the quantity scored in the Monte Carlo simulations is the energy deposition in a region of $1 \mathrm{~cm} \times 1 \mathrm{~cm}$ around the beam axis. The corresponding values of TPR 20,10 are given in the insets of panel (a) 
extracting region are of about $9 \%$ and $4 \%$ for $\bar{E}_{\Phi}$ and $\bar{E}_{\Psi}$, respectively, whereas for the bin width they are approximately $25 \%$ and $38 \%$, respectively. The influence of the size of the extracting region could be inferred from the spectra in Fig. 4, which for the 10 MV beam shows a clear shift of the most probable energy towards higher values for small extraction regions. This is consistent with the 3-D spatial energy distributions of the 6 and 10 MV PSFs, as that for $10 \mathrm{MV}$ shows a curvature that corresponds to higher energies at the beam center than in the periphery. For this reason, the spectra tabulated in the Additional file 1: Appendix are extracted from the respective PSFs for the entire field size, $10 \mathrm{~cm} \times 10 \mathrm{~cm}$ and $15 \mathrm{~cm} \times 15 \mathrm{~cm}$ for photons and electrons, respectively.

In spite of the differences mentioned, the mean photon energy of a MV spectrum is not a parameter used in reference dosimetry, as beams with the same mean energy might have different penetration properties (as it occurs, for example, with $\mathrm{kV} x$-ray beams). Hence, it is of interest to verify how the beam quality index $\mathrm{TPR}_{20,10}$ for a $10 \mathrm{~cm} \times 10 \mathrm{~cm}$ field, calculated with the spectra in the central region (e.g., $2 \mathrm{~cm} \times 2 \mathrm{~cm}$ around the beam central axis) and in the entire field size, compare with the value obtained using the PSF. The rationale for this comparison is that spectra used for the simulation of divergent beams, where a point source emitting the spectrum irradiates the solid angle subtended by the field size, neglect the correlation between energy, position and direction of the incident photons. The correlation is thus ignored in both spectra but is taken into account when the PSF is used, which in addition includes contaminant electrons and positrons. Depth-dose distributions for this analysis are shown in Fig. 5 for 6 and 10 MV, where the respective $\mathrm{TPR}_{20,10}$ values are indicated in the insets. These have been obtained from exponential fits between $5 \mathrm{~cm}$ and $25 \mathrm{~cm}$ depth, yielding $\mathrm{PDD}_{20,10}$, and using the empirical relation between $\mathrm{TPR}_{20,10}$ and $\mathrm{PDD}_{20,10}$ given in IAEA TRS-398 [4]. It was found that, for the small region spectra, the $\mathrm{TPR}_{20,10}$ values differ by $0.8 \%$ and $0.9 \%$ for 6 and $10 \mathrm{MV}$, respectively. For the entire field spectra, the differences were $1.2 \%$ and $1.9 \%$, respectively. However, considering for example a Farmer-type NE-2571 ionization chamber, these $\mathrm{TPR}_{20,10}$ correspond to $k_{Q}$ differences between $-0.06 \%$ and $-0.25 \%$ relative to those for the PSF, which being considerably smaller than the standard uncertainty of $k_{Q}(1 \%)$ can be considered to be negligible for $M V$ reference dosimetry. Figure $5 b$ shows the minor effect of neglecting the correlation between energy, position and direction of the incident photon spectra, where a small difference in the height of the maximum doses and their depths can be noted.

For electron beam spectra a detailed comparison is not feasible due to the lack of data. However, spectra from the internal report by DR [15] mentioned in the introduction have been obtained. They had been extracted from PSFs calculated with EGS4/BEAM and are compared to those in the present work in Fig. 6.

It should be stressed, however, that the DR data do not correspond to a conventional Varian linac, as the particular Clinac $2100 \mathrm{C}$ simulated in their study had thicker scattering foils and monitor chamber walls in order to match the depth-dose distributions of an earlier clinical

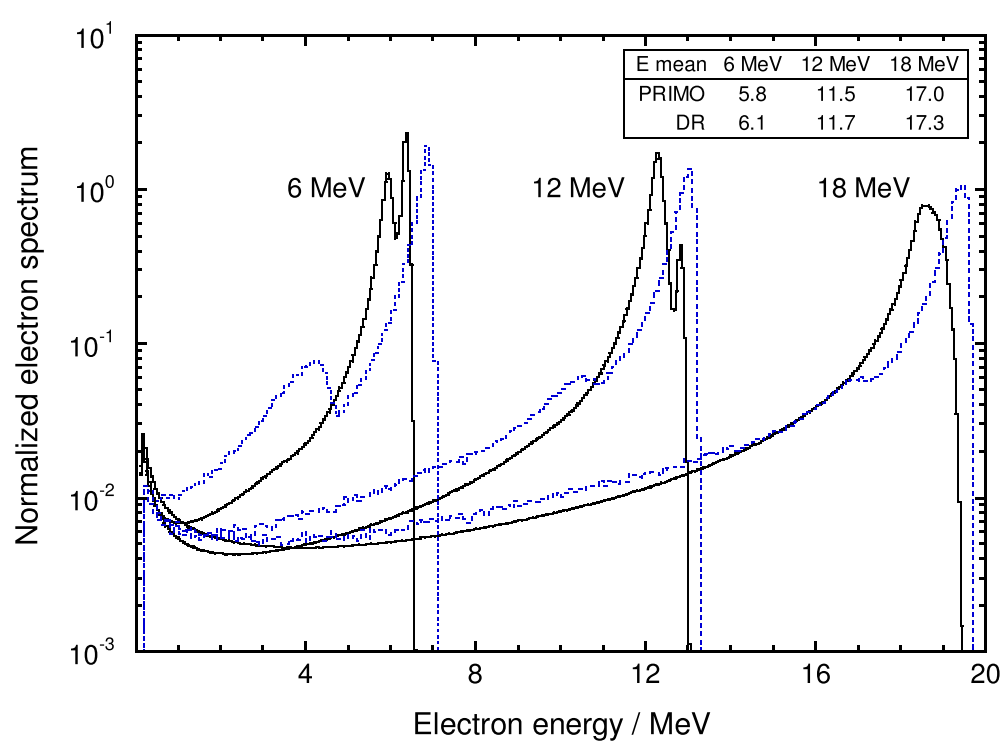

Fig. 6 Comparison of the PENELOPE/PRIMO electron spectra for Varian beams of 6, 12 and $18 \mathrm{MeV}$ with those calculated by Ding and Rogers [15], dotted histograms. All the spectra are normalized to their respective integral. The fluence-weighted mean energies of each spectrum are indicated in the inset. Note that the two linacs were not identical (see text) 
machine (in Wisconsin). Further, for the energies compared in Fig. 6, their field size was $10 \mathrm{~cm} \times 10 \mathrm{~cm}$, while $15 \mathrm{~cm} \times 15 \mathrm{~cm}$ was used in the present work. It can be observed in the figure that the two spectra datasets differ substantially but, from the comments above, significant differences were anticipated as the two linacs were not identical.

The conditions under which a spectrum is used in a $\mathrm{MC}$ calculation have also been analyzed for a $6 \mathrm{MeV}$ electron beam. As PSFs are tallied at the downstream end of the electron applicator, the simulation includes the air between the phantom surface and the electron applicator. Hence, a spectrum derived from the PSF includes the effect of air filtration. Sometimes, however, this condition is not properly accounted for and a point source is simulated at a certain SSD with air filling the space between the source and the phantom. This produces a double counting of the air effect, whose filtration can be of significance. Figure 7 compares the full PSF depth-dose distribution with those obtained using the spectrum under various configurations. The effect of an added $100 \mathrm{~cm}$ air filtration produces a less penetrating beam, which yields an incorrect depth-dose distribution. Using vacuum filling the space between the source and the phantom, or $95 \mathrm{~cm}$ vacuum followed by $5 \mathrm{~cm}$ of air (which is closer to the geometry for tallying the PSF), differs only by a few tenths of a percent from the PSF beam quality index $R_{50}$ and practical range $R_{\mathrm{p}}$, the results for both geometries being indistinguishable. The region in front of the depth-dose maximum differs, however, from that for the PSF because the correlation between energy, position and direction of every particle, as well as contaminating particles, are ignored in an incident electron spectrum.

\section{Conclusions}

An up-to-date set of consistent photon and electron spectra for a range of Varian accelerators has been calculated using the PENELOPE/PRIMO MC system. They have been extracted from PSFs calculated for field sizes of $10 \mathrm{~cm} \times 10 \mathrm{~cm}$ and $15 \mathrm{~cm} \times 15 \mathrm{~cm}$ for photon and electron beams, respectively, at an SSD of $100 \mathrm{~cm}$ using a bin width of $50 \mathrm{keV}$. Their use is intended for the simulation of detector-related quantities in conventional broad beams, where the use of detailed PSFs is less critical than for Monte Carlo-based treatment planning applications, but unlike PSFs, spectra can easily be transferred to other computer systems and users. They are provided in detailed tables and made available in digital form at the PRIMO web site for easy retrieval.

Spectra from this work have been compared to those obtained by other authors, showing rather good agreement with those calculated with the, now superseded, EGS4/BEAM MC system, but significant differences with the widely used "classic" photon data set from Mohan et al. (1985), available in the EGSnrc distribution package. Other spectra sets, particularly for electron beams, have not been previously published for the machines simulated in this work.

The influence of the bin width of the spectra extracted from the PSF has been investigated for 6 and $10 \mathrm{MV}$ photon spectra using 1, 50 and $250 \mathrm{keV}$ widths. Their impact on the fluence-weighted and energy fluence-weighted

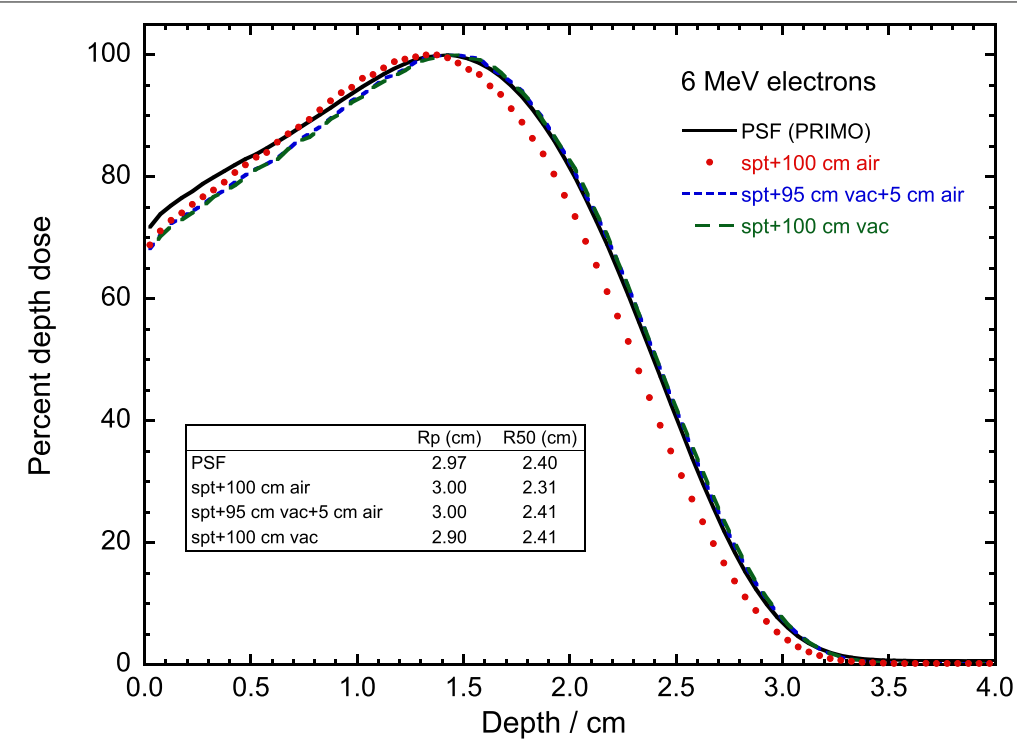

Fig. 7 Comparison of $6 \mathrm{MeV}$ electrons depth-dose distribution for a $15 \mathrm{~cm} \times 15 \mathrm{~cm}$ field, obtained from the PSF (solid line) with those obtained using the spectrum plus an added $100 \mathrm{~cm}$ air filtration (dots), $100 \mathrm{~cm}$ of vacuum filling the space between the source and the phantom (long dashes), and $95 \mathrm{~cm}$ vacuum followed by $5 \mathrm{~cm}$ of air (short dashes). The corresponding values of $R_{50}$ and $R_{p}$ are given in the inset 
mean energies, $\bar{E}_{\Phi}$ and $\bar{E}_{\Psi}$, respectively, has been found to be negligible. The effect of the size of the region around the beam central axis used to extract the spectra, a narrow zone or the entire beam size, has been analyzed for these beams. For $6 \mathrm{MV}$, differences of about $4 \%$ and $2 \%$ for $\bar{E}_{\Phi}$ and $\bar{E}_{\Psi}$, respectively, have been found; the differences become $25 \%$ and $38 \%$ for the $10 \mathrm{MV}$ beam. A comparison between depth-dose distributions for a $10 \mathrm{~cm} \times 10 \mathrm{~cm}$ field calculated with these spectra and those obtained from the PSFs yields differences in $\mathrm{TPR}_{20,10}$ values between $0.7 \%$ and $1.9 \%$. However, these correspond to negligible differences (up to $0.25 \%$ ) in the $k_{Q}$ values for a Farmer-type NE-2571 ionization chamber. The effect of disregarding the correlation between energy, position and direction in the incident photon spectra is rather small. It could be of some importance for distributions normalized at the depth of the maximum dose (depth doses or tissue-maximum ratios) due to the minor difference in the height and depth of the maxima, but is irrelevant for TPR distributions.

Different configurations for using electron spectra have been compared for $6 \mathrm{MeV}$ beams, concluding that the geometry used for tallying the PSFs used to extract the spectra must be accounted for in subsequent calculations using the spectra as a source. The jeopardy of over counting the influence of air filtration has been emphasized.

\section{Additional files}

Additional file 1: Table spectra.tex. The table is provided as a LaTeX file. Preferably it should be published as an Appendix; otherwise please make it available as an additional file in pdf. (TEX $60 \mathrm{~kb}$ )

Additional file 2: Table spectra.xlsx. The table is provided as an Excel "xlsx" file. It should be made available as an additional file in Excel format. (XLSX $123 \mathrm{~kb})$

\section{Abbreviations}

FFF: Flattening-filter-free; MC: Monte Carlo; PSF: Phase-space file; VRT: Variance-reduction technique

\section{Acknowledgments}

Not applicable.

\section{Funding}

The authors acknowledge support by the Open Access Publication Fund of the University of Duisburg-Essen. L Brualla, M Rodriguez and I Sempau were funded by the Deutsche Forschungsgemeinschaft (DFG) project BR 4043/3-1. J Sempau also acknowledges funding by H2020 EJP Concert, project 003-2017-PODIUM, and by the Spanish Networking Research Center CIBER-BBN.

\section{Availability of data and materials}

Data tables are available in digital form at the PRIMO web site (https://www. primoproject.net). The PRIMO system is also available at the same web site.

\section{Authors' contributions}

LB and PA conceived the study. LB conducted the simulations. PA analyzed and processed the data. MR coded the routines for PSF analysis. JS participated in the data analysis and contributed to draft the manuscript. All authors read and approved the final manuscript.
Ethics approval and consent to participate

Not applicable.

\section{Consent for publication}

Not applicable.

\section{Competing interests}

L Brualla, M Rodriguez and J Sempau are authors of the PRIMO system. $P$ Andreo declares that he has no competing interests.

\section{Publisher's Note}

Springer Nature remains neutral with regard to jurisdictional claims in published maps and institutional affiliations.

\section{Author details}

${ }^{1}$ West German Proton Therapy Centre Essen (WPE), D-45147 Essen, Germany. ${ }^{2}$ West German Cancer Center (WTZ), D-45147 Essen, Germany. ${ }^{3}$ University Hospital Essen, D-45147 Essen, Germany. ${ }^{4}$ Universität Duisburg-Essen, Medizinische Fakultät, D-45147 Essen, Germany. ${ }^{5}$ Centro Médico Paitilla, 0816-03075 Panama City, Panama. ${ }^{6}$ Instituto de Investigaciones Científicas y de Alta Tecnología, INDICASAT-AIP, City of Knowledge, Building 219, Panama City, Panama. ${ }^{7}$ Department of Physics and Institute of Energy Technologies, Universitat Politècnica de Catalunya, E-08028 Barcelona, Spain. ${ }^{8}$ Department of Medical Radiation Physics and Nuclear Medicine, Karolinska University Hospital, and Department of Oncology-Pathology, Karolinska Institutet, SE-171 76 Stockholm, Sweden.

Received: 1 August 2018 Accepted: 19 November 2018

Published online: 11 January 2019

\section{References}

1. Andreo P. Monte Carlo techniques in Medical Radiation Physics. Phys Med Biol. 1991:36:861-920.

2. Andreo P. Monte Carlo simulations in radiotherapy. Radiat Oncol. 2018;13:121.

3. Sempau J, Sánchez-Reyes A, Salvat F, Ouland-ben-Tahar H, Jiang SB, Fernández-Varea JM. Monte Carlo simulation of electron beams from an accelerator head using PENELOPE. Phys Med Biol. 2001;46:1163-86.

4. Andreo P, Burns DT, Hohlfeld K, Huq MS, Kanai T, Laitano F, Smyth VG, Vynckier S. Absorbed Dose Determination in External Beam Radiotherapy: An International Code of Practice for Dosimetry Based on Standards of Absorbed Dose to Water. IAEA Technical Reports Series no. 398. Vienna: International Atomic Energy Agency; 2000.

5. Palmans H, Andreo P, Huq MS, Seuntjens J, Christaki K. Dosimetry of Small Static Fields Used in External Beam Radiotherapy: An IAEA-AAPM International Code of Practice for Reference and Relative Dose Determination. IAEA Technical Report Series No. 483. Vienna: International Atomic Energy Agency; 2017.

6. Almond PR, Biggs PJ, Coursey BM, Hanson WF, Huq MS, Nath R, Rogers DWO. AAPM's TG-51 protocol for clinical reference dosimetry of high-energy photon and electron beams. Med Phys. 1999;26:1847-70.

7. McEwen M, DeWerd L, Ibbott G, Followill D, Rogers DWO, Seltzer S, Seuntjens J. Addendum to the AAPM's TG-51 protocol for clinical reference dosimetry of high-energy photon beams. Med Phys. 2014;41:041501-1-20.

8. Andreo P, Burns DT, Nahum AE, Seuntjens J, Attix FH. Fundamentals of lonizing Radiation Dosimetry. Weinheim, Germany: Wiley-VCH; 2017.

9. Czarnecki D, Poppe B, Zink K. Impact of new ICRU Report 90 recommendations on calculated correction factors for reference dosimetry. Phys Med Biol. 2018;63:155015.

10. Mohan R, Chui C, Lidofsky L. Energy and angular distributions of photons from medical linear accelerators. Med Phys. 1985;12:592-7.

11. Nelson WR, Hirayama H, Rogers DWO. The EGS4 Code System. Report SLAC 265. Standford, CA: Stanford Linear Accelerator Center; 1985.

12. Kawrakow I, Mainegra-Hing E, Rogers DWO, Tessier F, Walters BRB. The EGSnrc Code System: Monte Carlo Simulation of Electron and Photon Transport. NRCC Report PIRS-701. Ottawa: National Research Council Canada; 2017.

13. Sheikh-Bagheri D, Rogers DWO. Monte Carlo calculation of nine megavoltage photon beam spectra using the BEAM code. Med Phys. 2002;29:391-402 
14. Rogers DWO, Faddegon BA, Ding GX, Ma C-M, We J, Mackie TR. BEAM a Monte Carlo code to simulate radiotherapy treatment units. Med Phys. 1995;22:503-24.

15. Ding GX, Rogers DWO. Energy Spectra, Angular Spread and Dose Distributions of Electron Beams from Various Accelerators Used in Radiotherapy. NRCC Report PIRS-439. Ottawa: National Research Council Canada; 1995.

16. Capote R, Jeraj R, Ma C-M, Rogers DWO, Sánchez-Doblado F, Sempau J, Seuntjens J, Siebers JV. Phase-Space Database for External Beam Radiotherapy. IAEA Report INDC(NDS)-0484. Vienna: International Atomic Energy Agency; 2006.

17. Salvat F, Fernández-Varea JM, Sempau J. PENELOPE: A Code System for Monte Carlo Simulation of Electron and Photon Transport. Report NEA/NSC/DOC(2011). Issy-les-Moulineaux, FRA: OECD Nuclear Energy Agency; 2011.

18. Rodríguez M, Sempau J, Brualla L. PRIMO - A graphical environment for the Monte Carlo simulation of Varian and Elekta linacs. Strahlenther Onkol. 2013;10:881-6.

19. Brualla L, Rodríguez M, Sempau J. PRIMO User's Manual Version 0.3.1.1600. Strahlenklinik, Hufelandstrasse 55, D-45122 Essen, Germany: Universität Duisburg-Essen, Universitätsklinikum Essen; 2018.

20. Salvat F. PENELOPE-2014: A Code System for Monte Carlo Simulation of Electron and Photon Transport. Report NEA/NSC/DOC(2014). Issy-les-Moulineaux, FRA: OECD Nuclear Energy Agency; 2014.

21. Rodríquez M. PENLINAC - extending the capabilities of the Monte Carlo code PENELOPE for the simulation of therapeutic beams. Phys Med Biol. 2008;53:4573-93.

22. Sempau J, Badal A, Brualla L. A PENELOPE-based system for the automated Monte Carlo simulation of clinacs and voxelized geometries. Med Phys. 2011;38:5887-95.

23. Rodríguez M, Sempau J, Fogliata A, Cozzi L, Sauerwein W, Brualla L. A geometrical model for the Monte Carlo simulation of the TrueBeam linac. Phys Med Biol. 2015;60:219-29.

24. Rodríguez M, Sempau J, Brualla L. A combined approach of variance-reduction techniques for the efficient Monte Carlo simulation of linacs. Phys Med Biol. 2012;57:3013-24.

25. Brualla L, W S. On the efficiency of azimuthal and rotational splitting for Monte Carlo simulation of clinical linear accelerators. Rad Phys Chem. 2010;79:929-32.

26. Andreo P. The physics of small megavoltage photon beam dosimetry. Radioth Oncol. 2018;126:205-13.

27. Chibani O, Ma C-M. On the discrepancies between Monte Carlo dose calculations and measurements for the $18 \mathrm{MV}$ Varian photon beam. Med Phys. 2007;34:1206-16.

Ready to submit your research? Choose BMC and benefit from:

- fast, convenient online submission

- thorough peer review by experienced researchers in your field

- rapid publication on acceptance

- support for research data, including large and complex data types

- gold Open Access which fosters wider collaboration and increased citations

- maximum visibility for your research: over 100M website views per year

At $\mathrm{BMC}$, research is always in progress.

Learn more biomedcentral.com/submissions 